\title{
Conception et édition de jeux de société : étude du secteur (de niche) et trajectoires professionnelles
}

Designing and publishing board games: a study of the (niche) sector and professional trajectories

\section{Vincent Berry et Nathalie Roucous}

\section{OpenEdition}

\section{Journals}

Édition électronique

URL : http://journals.openedition.org/sdj/2732

DOI : $10.4000 /$ sdj.2732

ISSN : 2269-2657

\section{Éditeur}

Laboratoire EXPERICE - Centre de Recherche Interuniversitaire Expérience Ressources Culturelles Education

Référence électronique

Vincent Berry et Nathalie Roucous, « Conception et édition de jeux de société : étude du secteur (de niche) et trajectoires professionnelles », Sciences du jeu [En ligne], 13 | 2020, mis en ligne le 17 novembre 2020, consulté le 21 janvier 2021. URL : http://journals.openedition.org/sdj/2732 ; DOI https://doi.org/10.4000/sdj.2732

Ce document a été généré automatiquement le 21 janvier 2021.

\section{(i) $\$($

La revue Sciences du jeu est mise à disposition selon les termes de la Licence Creative Commons Attribution - Pas d'Utilisation Commerciale - Pas de Modification 4.0 International. 


\title{
Conception et édition de jeux de société : étude du secteur (de niche) et trajectoires professionnelles
}

\author{
Designing and publishing board games: a study of the (niche) sector and \\ professional trajectories
}

Vincent Berry et Nathalie Roucous

Un jeu de société, c'est bien plus qu'un « jouet avec des règles ». C'est une expérience humaine vécue par des joueurs, à la manière d'un livre,

d'un film, ou d'une pièce de théâtre dont les joueurs seraient les acteurs, et nous le metteur en scène. Nous ne sommes ni « inventeurs » ni « créateurs » : nous sommes auteurs et autrices

d'œuvres de l'esprit

(Extrait du manifeste de la société des auteurs de jeux de société, 2017)

Dans le cadre d'une enquête menée au sein du Master sciences du jeu et poursuivie au sein du programme de recherche "le Modèle Français", soutenu et financé par le Labex ICCA, nous nous sommes intéressés aux parcours des éditeurs et des auteurs de jeu de société. Il s'agissait d'identifier les différents acteurs à l'œuvre dans le processus de production d'un jeu, tout en s'intéressant à leurs trajectoires sociales, scolaires, professionnelles. Comment devient-on auteur et éditeur de jeu de société ? De quelle nature relèvent leurs activités ? S'agit-il d'un travail à plein temps ? Perçoivent-ils tout ou une partie de leur activité comme un » vrai boulot » (Bidet, 2011)?

À travers ces différents questionnements, il s'agit également d'esquisser les contours de ce « monde du jeu » (Berry, Borzakian, 2015), terme qui conduit, à la suite des travaux de Becker sur les "mondes de l'art", à étudier le "réseau de tous ceux dont les activités coordonnées grâce à une connaissance commune des moyens conventionnels de travail concourent à la production des œuvres » (Becker, 1988, p. 22), ici des jeux de 
société. Quelles sont les " chaines de coopération », les «modes d'interactions ", mais aussi les oppositions, les conflits et les règles (Bourdieu, 1998) qui structurent ce champ social, professionnel et culturel?

3 À un premier et rapide niveau d'analyse, le monde du jeu de société partage un certain nombre de propriétés communes avec d'autres secteurs artistiques, celui de la bande dessinée par exemple, de la littérature ou encore de la musique. Comparables à d'autres industries culturelles, les opérations de production y sont rationnalisées (Berthou, 2010), en partie standardisées, et une division du travail distingue «les auteurs », des " éditeurs ", des «illustrateurs", des "directeurs artistiques ». Dans le même temps, toute une économie symbolique, en termes de prestige et de réputation, structure une partie de l'espace : des prix viennent récompenser des auteurs et des titres. Une presse spécialisée analyse les sorties, en consacre certaines et propose des interviews avec des "professionnels". Les festivals sont nombreux et plusieurs salons internationaux dédiés au jeu de société sont organisés annuellement.

4 Si le monde du jeu de société présente ainsi certains traits d'un "monde de l'art », son inscription dans le domaine culturel demeure cependant ambigüe. Contrairement au monde musical ou littéraire dans lesquels l'intervention de l'état peut se traduire par le soutien d'une partie du secteur, la musique contemporaine par exemple (Menger, 1983), par des dispositifs de formation et professionnalisation, tels que les conservatoires pour la musique classique ou le jazz (Coulangeon, 1999), ou encore par un régime d'allocations spécifiques, le jeu de société demeure relativement invisible: les taux de TVA ${ }^{1}$ appliqués au jeu de société divergent (bénéficiant parfois de ceux du livre, mais pas toujours), le statut d'auteur de jeu n'a pas de reconnaissance administrative claire, et peu de dispositifs professionnels ou financiers ne viennent soutenir ou organiser le secteur.

5 A travers l'étude de quelques-uns de ses professionnels, il s'agit d'étudier l'organisation et la relation du jeu de société avec d'autres secteurs culturels, mais aussi d'apporter, par un terrain nouveau, des éléments de réflexion plus larges sur la professionnalisation des activités artistiques amatrices ou professionnelles qui constituent depuis longtemps un axe important des recherches en sociologie (Stiénon, 2008). Écrivains (Lahire et Bois, 2006), musiciens (Coulangeon, 2004), ou encore auteurs de BD (Boltanski, 1975 ; Piette, 2015) sont l'objet de nombreux travaux. A l'exception du jeu vidéo (Benghozi, Chantepie, 2017), les professionnels du secteur ludique en France restent peu étudiés ${ }^{2}$. On pourrait certes les considérer comme hors "de l'économie de la culture» (Benhamou, 2011) en les rattachant au monde du divertissement. Mais l'étude de leur activité révèle des problématiques et des questionnements tout à fait comparables à d'autres domaines culturels et artistiques plus légitimes : l'existence d'un régime d'auctorialité, l'opposition entre amateurs et professionnels, ou encore la dichotomie entre « vrai métier » et loisir.

\section{Quelques précisions méthodologiques}

D'un point de vue méthodologique, cette enquête s'appuie sur l'analyse de 35 entretiens. 18 ont été menés auprès d'auteurs de jeux ${ }^{3}$ et 17 auprès de professionnels liés aux métiers de l'édition: directeurs artistiques, graphistes, chefs de projets, chefs de produits, commerciaux et illustrateurs ${ }^{4}$. Parmi ces derniers, 12 sont à l'origine de la création de la maison d'édition et occupent, au moment de l'entretien, la fonction de 
gérant ou de co-gérant. Notre échantillon a ciblé les acteurs français 5 , à l'exception d'un éditeur allemand, d'un éditeur grec et d'un éditeur belge, mais dont les jeux sont distribués en France via des filiales ou par l'intermédiaire d'un autre éditeur français.

Nous nous sommes attachés à une certaine diversité en termes de poids des acteurs: "gros» et "petits» éditeurs, auteurs de jeux "débutants » ou "confirmés » en prenant comme indicateurs le nombre de jeux édités, le nombre de récompenses reçues, le mode de distribution (boutique spécialisée et/ou grande surface), la traduction d'un ou plusieurs jeux à l'international, la réédition d'un ou plusieurs titres et enfin la taille des équipes dans le cas des éditeurs. Les enquêtés ont été recrutés soit par mail, en mobilisant le réseau des étudiants du Master Sciences du jeu, soit à l'occasion du festival international des jeux à Cannes (2018, 2019 et 2020).

8 Concernant le secteur de l'édition, l'échantillon se compose en majorité d'éditeurs « de niche » (12 sur 18), entendus ici comme des petites équipes (entre 1 et 10 salariés) qui gèrent un catalogue restreint à une dizaine de jeux (en moyenne) qui sont distribués principalement dans les boutiques spécialisées. Six éditeurs relèvent plus directement du secteur de masse avec des équipes de salariés plus importantes (entre 11 et 30), des catalogues de plusieurs centaines de jeux, dont certains diffusés en grande surface et dont le développement international est important. Concernant les auteurs, huit peuvent être qualifiés de "confirmés »: plus de dix de leurs jeux ont été édités et certains ont été traduits à l'étranger et/ou récompensés par des prix.

9 Les distinctions que nous opérons en amont de la recherche sont bien sûr discutables. De « jeunes » auteurs ou de « petits » éditeurs peuvent en effet avoir peu de jeux à leur actif, mais largement récompensés et vendus. A l'inverse, d'autres peuvent avoir de nombreuses publications, mais qui restent relativement confidentielles. Les différences entre "confirmé " et "débutant ", "de masse " et "de niche » sont à considérer comme une première catégorisation que l'on retrouve dans les discours des acteurs, mais pas nécessairement sous cette seule forme d'indicateurs chiffrés. Les prix et le nombre d'exemplaires vendus occupent une place importante, les récompenses également, mais aussi le capital symbolique (la réputation), le capital social (la connaissance des acteurs de ce monde professionnel) et un certain «style » de jeu produit par un auteur ou un éditeur et reconnu comme distinctif par le public. Nous y reviendrons.

Deux grilles d'entretiens ont été élaborées, une première à l'attention des éditeurs, une seconde à l'attention des auteurs. Afin d'éviter des discours très généraux sur le jeu de société, il s'agissait de mener les entretiens à partir d'études de cas concrets, pour évoquer un jeu précis de sa conception à sa parution, avant d'ouvrir sur des questions plus transversales ${ }^{6}$. Cette démarche a nécessité que les enquêteurs se familiarisent préalablement à chaque entretien avec le catalogue de jeux de l'éditeur ou avec la production de l'auteur. Ce faisant, ils disposaient d'une connaissance des mécanismes, des thèmes et des règles qui leur permettaient d'être plus à même de saisir, dans leurs nuances, les processus de conception évoqués par les interlocuteurs en relation avec le design matériel, technique et ludique du jeu.

11 Précisons enfin que notre échantillon a été constitué de manière inductive, à partir des salons professionnels et de notre connaissance du champ. Sa représentativité reste difficile à établir, essentiellement parce que, en dehors de données professionnelles, ce secteur est méconnu aussi bien du champ académique que des statistiques publiques. Le monde professionnel ${ }^{7}$ estime ainsi à plus d'une centaine le nombre d'éditeurs en 
France en ne considérant que les éditeurs « exclusifs ». L'identification du métier est en effet complexe car des éditeurs dans d'autres domaines, le livre jeunesse par exemple, peuvent éditer des jeux de façon ponctuelle ou régulière. Une moyenne de salariés est tout aussi difficile à établir tant les données sont fragiles et les écarts de taille sont grands: entre une personne et une soixantaine de salariés pour les plus grandes structures, si l'on raisonne uniquement en termes de «maison d'édition » et de « filière nationale $»^{8}$.

Du côté des auteurs, à l'exception de l'enquête de la Société des Auteurs de Jeux (SAJ) ${ }^{9}$, les données sont aussi à construire. On estime à plus d'une centaine d'auteurs (ayant publié au moins un jeu dans les trois dernières années), mais le chiffre reste fragile tant l'activité est, nous le verrons, intermittente. Internet encourage par ailleurs de jeunes auteurs à développer leurs jeux par le biais du financement participatif, mais également par la diffusion de créations gratuites ou en autoédition sous le format de " jeux à imprimer ", plus connu à travers l'expression anglaise de Print and Play. Notons enfin qu'une partie du secteur, celui dit "de masse», n'a pas de "régime d'auctorialité » (Neeman, 2012) : les jeux ne sont pas nécessairement signés par un auteur et le droit d'auteur ne s'y applique pas.

S'il est difficile de rapporter notre échantillon à une population connue ${ }^{10}$, nous avons été attentifs à une certaine diversité des profils : tailles des équipes, fonctions occupées, ancienneté et expérience dans le milieu. La diversité en termes de genre est faible dans notre échantillon qui se révèle très masculin, aussi bien chez les éditeurs que chez les auteurs. Si cette répartition témoigne d'une réalité du milieu étudiép ${ }^{11}$ les vagues d'entretiens à venir s'attacheront à réduire cet écart. Cet article mobilise en effet des données et des premiers résultats d'une enquête toujours en cours.

\section{Auteurs de jeux : un deuxième métier}

Sans pouvoir généraliser sur le métier d'auteur, on peut constater qu'il s'agit de personnes relativement jeunes, autour de 44 ans, souvent diplômées (bac +5$)$, de niveau cadre ou profession intermédiaire ${ }^{12}$. Lorsque l'on regarde plus en détail les trajectoires scolaires et les parcours professionnels, les domaines scientifiques, économiques et techniques prédominent sur les études artistiques, littéraires et sur les sciences sociales. Par ailleurs, comme l'indique le tableau 1, le travail d'auteur à temps plein est minoritaire chez nos enquêtés. Sur les 17 auteurs interrogés, seuls 6 d'entre eux considèrent cette activité comme leur principal métier. Le sentiment d'être " auteur " naît le plus souvent avec la première publication d'un jeu dans une société « reconnue $»^{13}$ : » Pour un jeune auteur, signer son premier jeu chez un éditeur connu, ouah! C'est le Graal!» (Thomas, auteur). Auparavant sont évoquées des pratiques d'« amateur ", des jeux "bricolés » pour être joués dans le cercle familial ou amical, sans visée éditoriale ni diffusion commerciale.

Pour l'écrasante majorité d'entre eux, les auteurs de jeux exercent donc un «second métier» rattaché aux professions intermédiaires: enseignement, animation socioculturelle, santé, informatique, journalisme, droit ou ingénierie ${ }^{14}$. La conception occupe un temps important pour quelques-uns. Pour d'autres, plus nombreux, c'est une activité qui, comme chez les écrivains (Lahire et Bois, 2006), se révèle « intermittente »: «Je suis prof de mathématiques en lycée, depuis un peu plus de 15 
ans maintenant. Je suis auteur de jeu à temps très très partiel, depuis 3 ans, date à laquelle le premier jeu a été publié » (Sylvain, auteur).

Intermittente, l'activité de création de jeu est également peu rémunératrice et impose le plus souvent d'autres sources de revenus. Comparable en cela à la situation des écrivains ${ }^{15}$ (Sapiro et Rabot, 2017), des musiciens ${ }^{16}$ (Buscatto, 2004) ou des auteurs de bandes dessinées ${ }^{17}$ (Groensteen, 1999) il s'agit, comme l'explique Dominique, d'assurer les fins de mois : " quand tu es auteur, tu gagnes pas des milles et des cents. Tu gagnes des centimes par boites vendues, t'as intérêt de bosser à côté si tu veux bouffer ». Si cette réalité économique est maintes fois rappelée par les professionnels et par la presse, les millions d'exemplaires vendus du Trivial Pursuit (Hasbro, 1984), du Pictionary (Parker, 1985) ou des Loups-Garous de Thiercelieux (Lui-même, 2001) ne manquent cependant pas d'être évoqués dans les discours des enquêtés, à titre de modèles ou d'exceptions notables (mais toujours désirables) qui masquent parfois l'ordinaire du métier aux yeux du public.

Tableau 1. Profil des auteurs interrogés ${ }^{18}$

\begin{tabular}{|c|c|c|c|c|c|c|c|c|}
\hline Prénom & Age & Formation & $\begin{array}{l}\text { Activité } \\
\text { principale }\end{array}$ & $\begin{array}{l}\text { Métiers } \\
\text { Passés }\end{array}$ & \begin{tabular}{|c|} 
Nbre de \\
jeux édités
\end{tabular} & $\begin{array}{c}\text { Jeux } \\
\text { primés }\end{array}$ & Réédition & \begin{tabular}{|l|} 
Jeux traduits à \\
linternational
\end{tabular} \\
\hline Xavier & 39 & Baccalauréat & \begin{tabular}{|l|} 
Ludothécaire \\
\end{tabular} & $\mathrm{N} / \mathrm{A}$ & 3 & Non & Non & \begin{tabular}{|l|} 
Oui \\
\end{tabular} \\
\hline Yves: & 42 & $\begin{array}{l}\text { Animation } \\
\text { socioculturelle } \\
\text { (licence pro.) }\end{array}$ & \begin{tabular}{|l}
$\begin{array}{l}\text { Auteur, } \\
\text { formateur } \\
\text { (Equel design) }\end{array}$
\end{tabular} & $\begin{array}{l}\text { Ludothécaire, } \\
\text { gamer } \\
\text { designer (jeu } \\
\text { vidéo) }\end{array}$ & $\begin{array}{l}2 \text { jeux, } 12 \\
\text { extensions }\end{array}$ & Non & Oui & Oui \\
\hline Richard & 35 & $\begin{array}{l}\text { Informatique } \\
\text { (Licence } \\
\text { Professionnelle) }\end{array}$ & Informaticien & $\begin{array}{l}\text { Educateur } \\
\text { Sportif }\end{array}$ & 4 & Non & $\underline{\underline{\text { oui }}}$ & Oui \\
\hline Dominique & 52 & $\begin{array}{l}\text { Communication, } \\
\text { publicité } \\
\text { (Master) }\end{array}$ & $\begin{array}{l}\text { Journaliste } \\
\text { (tiélévision, } \\
\text { information et } \\
\text { documentaire) }\end{array}$ & N/A & 9 & Non & ㅇi & Oui \\
\hline Sylvain & 48 & $\begin{array}{l}\text { Mathématiques } \\
\text { (Master) }\end{array}$ & $\begin{array}{l}\text { Professeur de } \\
\text { Mathématique } \\
5\end{array}$ & N/A & 15 & Non & $\underline{\text { non }}$ & Oui \\
\hline Quentin & 43 & $\begin{array}{l}\text { Étude de droit } \\
\text { (master) }\end{array}$ & \begin{tabular}{|l|}
$\begin{array}{l}\text { Conseiller } \\
\text { juridique }\end{array}$ \\
\end{tabular} & N/A & 16 & Non & Non & Oui \\
\hline Benoit & 42 & \begin{tabular}{|l|}
$\begin{array}{l}\text { Maitrise } \\
\text { d'histoire }\end{array}$ \\
\end{tabular} & \begin{tabular}{|l|}
$\begin{array}{l}\text { Profeesseur } \\
\text { d'histoire }\end{array}$ \\
\end{tabular} & N/A & 5 & Non & Oui & Non \\
\hline Franck & 53 & $\begin{array}{l}\text { Communication } \\
\text { (BTS) }\end{array}$ & \begin{tabular}{|l|} 
Auteur, \\
formateur \\
(game design) \\
\end{tabular} & $\begin{array}{l}\text { Maquettiste } \\
\text { PAO } \\
\text { (publicitíé) } \\
\end{array}$ & 3 & Oui & Oui & Oui \\
\hline Marc & 55 & Études de Santé & \begin{tabular}{|l|} 
Dentiste \\
\end{tabular} & N/A & 8 & Non & oui & Oui \\
\hline Renaud & 57 & Marketing (BTS) & Auteur & \begin{tabular}{|l}
$\begin{array}{l}\text { Dessinateur } \\
\text { industriel, } \\
\text { Huissier }\end{array}$ \\
\end{tabular} & 70 & Oui & Oui & Oui \\
\hline Lucas & 53 & $\begin{array}{l}\text { Ingénierie } \\
\text { mécanique } \\
\text { (Licence) }\end{array}$ & Auteur & $\begin{array}{l}\text { Concepteur } \\
\text { Produit }\end{array}$ & 4 & oui & Non & Oui \\
\hline Alexandre & 37 & $\begin{array}{l}\text { Sciences } \\
\text { Physiques } \\
\text { (Master) } \\
\end{array}$ & $\begin{array}{l}\text { Chargé d'étude } \\
\text { (marketing) }\end{array}$ & \begin{tabular}{|l|} 
Game \\
Designer \\
(jeux vidéo)
\end{tabular} & $\begin{array}{l}5 \text { jeux, } 11 \\
\text { extensions }\end{array}$ & Oui & Non & Oui \\
\hline Bertrand & 30 & $\begin{array}{l}\text { Managementent et } \\
\text { gestion de Projet } \\
\text { (Master) }\end{array}$ & $\begin{array}{l}\text { chef de Projet } \\
\text { multimédia }\end{array}$ & $\begin{array}{l}\text { Game } \\
\text { Designer } \\
\text { (jeux vidéo) }\end{array}$ & 3 & non & Non & $\underline{\text { oui }}$ \\
\hline Louis & 56 & $\begin{array}{l}\text { Doctorat en } \\
\text { histoire }\end{array}$ & \begin{tabular}{|l}
$\begin{array}{l}\text { Professeur en } \\
\text { lycée }\end{array}$ \\
\end{tabular} & $\mathrm{N} / \mathrm{A}$ & $60+$ & Oui & oui & Oui \\
\hline Thomas & 29 & $\begin{array}{l}\text { Master } \\
\text { Informatique }\end{array}$ & Ludothécaire & N/A & $\begin{array}{l}3(4 \\
\text { extensions } \\
\text { d'un jeu) }\end{array}$ & Oui & oui & Oui \\
\hline Olivier & 41 & $\begin{array}{l}\text { Statistiques } \\
\text { (Master) }\end{array}$ & $\begin{array}{l}\begin{array}{l}\text { Auteur et } \\
\text { Editeur }\end{array} \\
\end{array}$ & $\begin{array}{l}\text { Enseignant } \\
\text { (contractuel) }\end{array}$ & 17 & oui & Oui & oui \\
\hline Yohann & 40 & $\begin{array}{l}\text { Commerce et } \\
\text { management }\end{array}$ & \begin{tabular}{|l|} 
Auteur, \\
formateur \\
(game design), \\
formation en \\
entreprise \\
\end{tabular} & $\begin{array}{l}\text { Chef de } \\
\text { publicité, } \\
\text { animateur } \\
\text { scientifique, } \\
\text { game master }\end{array}$ & 1 & Non & Non & Oui \\
\hline
\end{tabular}

Même parmi les rares auteurs déclarant pouvoir vivre exclusivement de leur création, la plupart font cohabiter d'autres activités professionnelles avec celle de créateur de jeu. Certains auteurs à succès, comme Louis ou Marc, maintiennent une vie professionnelle relativement éloignée du monde du jeu. Pour d'autres en revanche, leur activité " d'auteur » est déclarée comme première, voire unique, mais elle est dans les faits, comme chez les musiciens ou les écrivains, associée à des activités connexes à celle de la création, sous forme d'auto-entreprenariat ou de micro-entreprise: formation, édition, conseil, enseignement autour du jeu et du game design, etc.

La première raison évoquée pour expliquer cette pluriactivité est bien sûr la possibilité ou la difficulté « d'en vivre » et le caractère incertain de leurs gains. Construit le plus 
souvent en France sur le modèle dit des " royalties » (un pourcentage est reversé sur la vente de chaque exemplaire $\left.{ }^{19}\right)$, le marché du jeu de société apporte rarement des ressources suffisantes, sauf bien sûr en cas de succès, ou encore quand la production d'un auteur est conséquente. Louis, auteur prolixe, capé et récompensé pour plusieurs de ses jeux, reçoit selon les années entre « 40000 et 100000 euros de royalties ». Ce cas est rare et l'ensemble des acteurs interrogés s'accordent à considérer qu'il existe en France entre 10 et 15 auteurs qui vivent ou qui pourraient vivre de leur droit d'auteur. Dans l'enquête de la SAJ (2019), plus de $60 \%$ des auteurs interrogés déclaraient avoir reçu moins 5000 euros de droits d'auteur pour leurs créations. ${ }^{20}$

\section{La (relative) valeur symbolique du métier}

Même si elle est majoritairement soulignée, la dimension financière n'est pas la seule raison convoquée pour expliquer le non-engagement total dans le "métier d'auteur ». D'abord, même si le terme est employé par les acteurs eux-mêmes, l'absence d'identité institutionnelle claire ( pas de fiche RNCP $»^{21}$ ) pose problème pour certaines démarches administratives comme la déclaration de revenus. Ensuite, d'autres peinent à utiliser les termes «de métier d'auteur " dès lors que l'activité de conception est un complément par rapport à un «vrai » travail. Ainsi, pour Marc, la création de jeu est " un loisir qui rapporte un peu quand même, mais cela reste un loisir ». Il cumule ses activités d'auteur (et d'éditeur) et son activité de dentiste. La " question d'arrêter » son activité médicale pour se consacrer à plein temps au jeu de société s'est posée à plusieurs reprises, mais il ne "se sent pas » de faire le pas pour préserver un statut social plus gratifiant et prestigieux. C'est en partie la même logique qui préside chez Louis, dont un de ses jeux s'est pourtant vendu à plus de 2 millions d'exemplaires. S'il reconnait pouvoir "vivre de ses droits d'auteurs", l'attachement à son métier d'enseignant l'emporte. D'une part, il s'agit d'un métier difficile, mais qui offre un «confort moral» et le sentiment d'une "utilité sociale». D'autre part, tout en défendant le métier d'auteur comme une activité professionnelle spécifique, l'activité lui semble plus « dilettante».

Au-delà de la seule nécessité économique, la valeur sociale et symbolique attachée au métier d'auteur de jeu ne suffit pas toujours à faire basculer dans une activité «à plein temps ", même en cas de succès. L'appartenance du jeu de société au monde du loisir et $\mathrm{du}$ frivole rend souvent peu audible, voire suspecte, la notion de métier. Comme l'explicite l'un d'entre eux, d'origine modeste, c'est la définition même de la notion de travail qui est en conflit avec celle de jeu. La contrainte et la souffrance propre au monde du « vrai » travail (ouvrier en l'occurrence) sont peu compatibles avec celle de plaisir propre au jeu.

Quand tu dis que t'es un créateur de jeu en France, quand t'expliques ça aux gens, ils comprennent pas ; ils s'imaginent pas ce que c'est ; ils pensent que tu t'amuses à la maison tout le temps. Ils se rendent pas compte que c'est un vrai travail. Mes parents sont d'un milieu ouvrier : mon père était ouvrier sur les chantiers, ma mère est femme de ménage. Si tu veux, pour eux, un travail, c'est un travail dans un bureau, c'est physique, des choses comme ça. (Renaud, auteur)

Du côté des auteurs qui ont basculé dans une activité d'« auteur à plein temps » (mais qui inclut diverses activités dans le domaine du jeu), la transition s'opère selon deux logiques : soit parce que leur activité professionnelle s'est arrêtée ou était en déclin, soit parce qu'ils ne pouvaient pas ou plus concilier leur vie professionnelle avec la 
création de jeux devenue plus intense. C'est le cas de Renaud qui, avant de consacrer sa vie professionnelle au jeu, travaillait dans l'administration fiscale ou encore de Franck qui après son licenciement a fait le choix de développer cette activité jusque-là marginale.

Si cette possibilité de « vivre » ou de « pouvoir vivre » du métier d'auteur se révèle très rare, elle marque cependant une étape importante dans la carrière et structure pour partie le monde du jeu entre ceux pour qui il s'agit de la « vraie vie » et les autres. Cette division est, nous le verrons, un des marqueurs dans les carrières qui participent, avec d'autres, des critères qui séparent symboliquement les "grands auteurs » des « autres » comme le précise Quentin:» On a rencontré pour la première fois des gens du milieu. À un moment donné, je me vois leur poser la question: "et sinon dans la vraie vie vous faites quoi ?" Les mecs m'ont regardé et là j'ai pris conscience que, eux, c'était leur vraie vie, en fait, de faire ce genre de choses".

À un premier niveau d'analyse, le métier d'auteur de jeu partage ainsi un certain nombre de traits communs avec d'autres mondes culturels. Outre le caractère précaire, intermittent et souvent peu rémunérateur de l'activité, on retrouve assez bien les «figures » que décrivent Lahire et Bois à propos des « manières d'être écrivain » (2006, p. 78) : ceux pour qui l'activité de création reste occasionnelle et secondaire vis-à-vis d'un métier («les occasionnels»); ceux qui investissent l'essentiel de leur temps sans pouvoir s'y consacrer entièrement («les mordus») et enfin ceux qui vivent de leur création et de pratiques connexes dans le domaine du jeu ("les professionnels»). Néanmoins, une différence majeure apparait : si le statut d'auteur de jeu est revendiqué par beaucoup, sa valeur sociale, juridique et symbolique reste floue. Le travail «à plein temps » n'est pas nécessairement désiré même parmi ceux qui, dans le monde du jeu, font partie des auteurs consacrés et qui pourraient en vivre ${ }^{22}$.

\section{Les éditeurs}

Du côté des éditeurs, les parcours et les trajectoires sont un peu différents. Également diplômés en grande majorité au niveau Bac+3 ou Bac +5 , mais plus jeunes (autour de 36 ans), on constate une présence plus importante des formations liées au marketing, au commerce et aux sciences de gestion. Contrairement aux auteurs, l'édition est bien plus souvent l'activité professionnelle principale. De fait, rares sont les éditeurs de notre échantillon qui cumulent deux emplois. Dans les récits de vie se dessine souvent une trajectoire «type »: travaillant dans des domaines professionnels divers, mais passionnés par ailleurs par le jeu, certains ont basculé dans le secteur, sous l'effet d'une conjonction entre une situation professionnelle difficile ou peu satisfaisante et l'opportunité d'une nouvelle carrière: "Avant j'étais directeur de création dans une agence de pub. Bernard, un ami, avait toujours des sociétés de jeux et lorsque ma boite allait pas trop bien, il m'a proposé m'associer avec lui et ne faire que du jeu. Maintenant ça va faire 15 ans » (Stéphane, auteur/éditeur). 
Tableau 2. Profil des éditeurs interrogés ${ }^{23}$

\begin{tabular}{|c|c|c|c|c|c|c|c|c|c|c|}
\hline & Age & Formation & Fonction & Métiers passés & \begin{tabular}{|l|}
$\begin{array}{l}\text { Nore jeux } \\
\text { édites }\end{array}$ \\
\end{tabular} & \begin{tabular}{|l|} 
Jeux à \\
linternational \\
\end{tabular} & Jeux primés & Nbre de salariés & $\begin{array}{l}\begin{array}{l}\text { Date } \\
\text { de création }\end{array} \\
\text { de con }\end{array}$ & (Co)Gérant \\
\hline Jérôme & 50 & $\begin{array}{l}\text { Ecole supérieure } \\
\text { d'art }\end{array}$ & \begin{tabular}{|l|} 
Directeur \\
atrisique, \\
Graphiste auteur
\end{tabular} & Directeur Artistique & \begin{tabular}{|l|l|l|l|l|l|l}
2 \\
\end{tabular} & Oui & Non & 3 & 2010 & oui \\
\hline Stéphane & 41 & \begin{tabular}{|l|} 
Graphisme/' \\
Communication \\
(BTS)
\end{tabular} & \begin{tabular}{|l} 
Directeur \\
artistique, \\
Graphiste auteur
\end{tabular} & $\begin{array}{l}\text { Directeur de créstion dans une agence de } \\
\text { publicité }\end{array}$ & 15 & Oui & Non & 4 & 2010 & Oui \\
\hline Jean & 32 & Vente (BTS) & Chef de projet & Commercial & 15 & Oui & Non & 5 & 2008 & Non \\
\hline Hector & 32 & \begin{tabular}{|l|} 
Economie \\
(Master)
\end{tabular} & Chef de projet & Auditeur Comptable & $1000+$ & Oui & Oui & 70 (filiale française) & 1883 & Non \\
\hline Fabrice & 43 & \begin{tabular}{|l}
$\begin{array}{l}\text { Géographie } \\
\text { (Master) }\end{array}$ \\
\end{tabular} & Gérant & $\begin{array}{l}\text { Directeur de librainie, Chef de projer digital, } \\
\text { directeur commercial (edition de jeux) }\end{array}$ & 10 & Oui & Oui & 1 & 2013 & oui \\
\hline Victor & 42 & \begin{tabular}{|l}
$\begin{array}{l}\text { Ecole de } \\
\text { commerce } \\
\text { (Master) }\end{array}$ \\
\end{tabular} & \begin{tabular}{|l} 
Directeur \\
Marketing
\end{tabular} & Gestiounaire de projet (accessories de cuisine) & $100+$ & Oui & Non & 12 & 1980 & oui \\
\hline Gilles & 36 & \begin{tabular}{|l|} 
Sciences \\
Physiques \\
(Master)
\end{tabular} & Chef de projet & Professseur de physique en lycée & 17 & Oui & Oui & 5 & 2010 & oui \\
\hline Alain & 31 & \begin{tabular}{|l} 
Polytechnique \\
oyle
\end{tabular} & Cbef de produit & Ingenieur & $300+$ & Oui & Oui & 30 & 2009 & oui \\
\hline Paul & 42 & \begin{tabular}{|l|} 
Architecture \\
\end{tabular} & \begin{tabular}{|l|l|l} 
Directeur artistique \\
\end{tabular} & Presse jeumesse & $200+$ & Oui & Oui & 12 & 2005 & Oui \\
\hline Théodore & 32 & \begin{tabular}{|l|} 
Exudes \\
Supérieures des \\
Techniques du \\
Son \\
\end{tabular} & Directeur artistique & \begin{tabular}{|l|} 
Ingénieut son \\
\end{tabular} & 3 & Oui & Non & 1 & 2014 & oui \\
\hline Benjamin & 30 & \begin{tabular}{|l|}
$\begin{array}{l}\text { Ecole de } \\
\text { commerce }\end{array}$ \\
\end{tabular} & $\begin{array}{l}\text { Aasistant chef de } \\
\text { produit }\end{array}$ & $\begin{array}{l}\text { Assistant Chef de Marché Livre, Assistant } \\
\text { Analyse Marketing }\end{array}$ & $200+$ & Oui & Oui & 15 & 1947 & Non \\
\hline Michael & 38 & \begin{tabular}{|l|} 
Sciences \\
économiques \\
(licence)
\end{tabular} & $\begin{array}{l}\text { Directeur de } \\
\text { publication }\end{array}$ & Gestioumaire de Licence, éditeur livres de SF & 29 & Non & Oui & 1 & & oui \\
\hline Thierry & 29 & \begin{tabular}{|l|} 
Sciences de \\
gestion (Master)
\end{tabular} & \begin{tabular}{|l} 
Responsable \\
Marketing chef de \\
produtit
\end{tabular} & Gestion de licence & \begin{tabular}{|l|}
15 jewr, 30 \\
extensions
\end{tabular} & Oui & Non & 7 & 2012 & Non \\
\hline Jonathan & 34 & $\begin{array}{l}\text { Marketing } \\
\text { (licence) }\end{array}$ & Directeur artistique & Imprimeur (employé) & 12 & Non & Non & 1 & 2012 & oui \\
\hline Sofien & 24 & \begin{tabular}{|l|} 
Design \\
Graphique \\
(BTS, école des \\
Gobelins) \\
\end{tabular} & Graphiste & N/A & \begin{tabular}{|l|}
11 jeur, 6 \\
entensions
\end{tabular} & Oui & Oui & 11 & 14 & Non \\
\hline $\begin{array}{l}\text { Yamnick } \\
\end{array}$ & 43 & \begin{tabular}{|l|} 
Baccalaurieast \\
(deux annees \\
histoire / \\
geograghie)
\end{tabular} & Directeur éditorial & Presse hodique, Libraire & 3 & Oui & Non & 3 & 2016 & Oui \\
\hline Eddy & 40 & \begin{tabular}{|l} 
Baccallauréat \\
STT
\end{tabular} & Graphiste & $\begin{array}{l}\text { Manutentiounaire, technicien en maintenance } \\
\text { informatique, employé salle de jeux en réseasu }\end{array}$ & \begin{tabular}{|l|}
15 jeur, 5 \\
extensions
\end{tabular} & Oui & Oui & 7 & 2011 & oui \\
\hline Anna & 32 & \begin{tabular}{l|} 
Métiers du linre \\
et de Pédition \\
(Master)
\end{tabular} & $\begin{array}{l}\text { Assistante } \\
\text { éditoriale }\end{array}$ & Secrétarire đéditition conseil général & \begin{tabular}{|l|}
16 jeur, 5 \\
entensions
\end{tabular} & Oui & Oui & 7 & 2011 & Non \\
\hline
\end{tabular}

Dans notre échantillon majoritairement composé d'éditeurs « de niche », la rhétorique de l'aventure entrepreneuriale est souvent mobilisée. Engagé dans une activité professionnelle, précaire, en déclin ou peu satisfaisante, le métier de l'édition permet de passer d'une intégration professionnelle «laborieuse ", "incertaine» ou "disqualifiante " à une intégration plus "assurée » pour reprendre les termes de Paugam (2017). Pour autant, la situation d'une grande partie de ces éditeurs reste fragile. Gilles, ancien enseignant, reconnait avoir quitté un métier stable, " épuisant " et peu gratifiant pour une activité plus précaire, moins rémunératrice mais en lien avec sa "passion ». Dans le cas de la société de Stéphane, les trois fondateurs, dont l'un a quitté sa fonction de cadre à Pôle Emploi, gagnent chacun à peu à près « un SMIC [5: travailler désormais dans un domaine qu'ils affectionnent. Ils s'estiment " chanceux » et évoquent " un métier idéal », « un super travail ».

Plus que chez les auteurs, la logique d'une compensation symbolique est donc présente. Le métier est précaire, exigeant, parfois aliénant, et la rentabilité, estimée par plusieurs autour de 10000 exemplaires vendus pour un jeu, n'est pas toujours au rendez-vous. Mais le travail apparait d'autant plus apprécié que l'activité d'édition se révèle éloignée de la profession précédemment exercée. De sorte que, si l'entrée dans le monde du jeu se présente toujours comme une réorientation et non comme une insertion professionnelle directe, elle prend des formes différentes. Dans le cas des auteurs, l'activité de conception reste compatible avec le maintien d'une autre activité, alors que pour les éditeurs l'activité prend le pas sur la précédente, comme une activité pleine et entière et non parallèle ou complémentaire à une autre. L'entrée se fait ainsi de façon plus marquée pour les éditeurs alors qu'elle se tisse au fil du temps pour les auteurs. Dans les deux cas, il y a une « histoire personnelle avec le jeu » à l'origine de leur « vocation". 


\section{Culture ludique et vocation} celui de la bande dessinée (Piette, 2015) ou à certains domaines musicaux (Coulangeon, 2004), auteurs et éditeurs se rejoignent pour mettre en avant leur passé de «passionné » comme déclencheur de leur vocation. Benoit, auteur de 5 titres, parle de sa "passion du jeu " à l'origine de ses créations : "c'est quelque chose que j'avais eue dans la plus jeune enfance bien que nous ne fussions pas une famille très joueuse.» Certains déclarent avoir été fortement influencés par un jeu en particulier ou un type de jeu qui a conditionné leur pratique de création, les jeux de rôle et les «Grandeur Nature » par exemple qui ont été dans la jeunesse de Quentin «fondateurs» de sa trajectoire d'auteur. Comme observé chez les musiciens de variétés, de rock ou de $\mathrm{jazz}^{24}$, les pratiques amateurs de l'adolescence apparaissent bien souvent comme «le préambule ordinaire de la professionnalisation » (Coulangeon, 2004). sont cités, les auteurs mentionnés, les règles et les mécanismes connus. Manifestant une culture ludique érudite, ils mettent en lien leur passé de joueur et leur activité de concepteur en rapport avec leur vocation. La relation entre pratiques enfantines et créations ludiques est une logique maintes fois évoquée dans les entretiens, rejoignant les analyses de Brougère (2008) sur le «recyclage » de la culture enfantine dans les produits ludiques contemporains. Marc établit un lien entre les jeux « amateurs » créés pour ses enfants et son activité de création: «j'ai toujours bricolé le bois quand mes enfants étaient petits. J'ai fait des jouets en bois pour Noël par exemple. Après lorsqu'ils étaient plus grands, je recopiais des jeux connus comme par exemple le Stratégo ».

Chez les éditeurs également, la passion du jeu est évoquée comme un facteur important dans leur choix de carrière. Pour Théodore, il s'agit d'une dimension centrale, voire nécessaire, pour le développement et la pérennité de l'entreprise : «On ne se lance pas dans une maison d'édition de jeux sans connaitre un minimum. Parce que sinon tu te plantes, c'est normal. » (Théodore, éditeur). Cette culture ludique ne se construit cependant pas seulement dans un rapport au passé car les auteurs et les éditeurs demeurent des joueurs réguliers : ils jouent pour tester leur propre création ou un projet d'édition bien sûr, mais aussi dans le cadre de leur loisir.

\section{Autodidaxie et effets de formation}

Dans les processus de professionnalisation décrits, la socialisation familiale et la formation scolaire sont rarement ou très indirectement évoquées, moins encore par les auteurs. Les pratiques ludiques passées et le "frayage» (Delbos et Jorion, 1990) apparaissent comme les principales formes sociales d'apprentissages du métier. Comparables à ce qu'observe la sociologie des amateurs (Stienon, 2008), un "goût personnel » et différentes formes d'autodidaxie sont souvent érigés à la fois comme principes explicatifs de leur vocation et comme moteur d'apprentissage du métier.

31 Si une solide culture ludique semble nécessaire, auteurs et éditeurs s'accordent à dire qu'il ne suffit pas d'être ou d'avoir été un «gros joueur». Des pratiques professionnelles peuvent agir directement sur leur vocation. Pour Benoît, professeur de 
lettres et auteur de jeux autour du langage, son activité de créateur est à mettre en lien avec celle d'enseignant : « je suis passionné de langues, il y en a que je connais et que je maitrise, d'autres que je découvre. Je suis surtout passionné par l'histoire des langues, mais surtout par les méthodes de les enseigner. » De la même façon, Bertrand, auteur spécialisé dans la création jeux de société « hybrides », ${ }^{25}$ travaille parallèlement en tant que chef de projet multimédia dans une société de jeu vidéo. Sans aucun doute possible, il voit dans ses créations les effets de sa carrière professionnelle. Comme analysé par Lahire et Bois (2006) chez les écrivains, à la variété des trajectoires sociales et des différents métiers exercés peut ainsi correspondre des "thèmes et des styles" de création.

Si, du côté des auteurs, la formation scolaire est rarement évoquée dans la vocation ou l'apprentissage du métier, elle est toujours présente en filigrane, moins dans les entretiens que dans les trajectoires. Le niveau scolaire et la surreprésentation des filières scientifiques chez les auteurs de jeu de société témoignent en effet d'un socle de connaissances et de compétences importantes, notamment dans le domaine des mathématiques et de la logique. Thomas, diplômé d'un master en informatique, estime nécessaire la compréhension des mathématiques pour l'« équilibrage » des règles, mais aussi une habitude de pensée dans la construction d'un système de règles qui permettent d'éviter les "bugs " ou "le King Making " ${ }^{26}$. Même chez les auteurs dont certains se considèrent comme littéraires » de formation » ou "de cœur ", « il faut un niveau de compréhension minimal en mathématiques. Ça ne fait pas tout, mais c'est important. Moi je me considère comme entre les deux » (Louis).

Du côté des éditeurs, le poids du monde scolaire dans la vocation est plus visible, à travers notamment l'évocation de « stages » exigés dans le cadre scolaire. Réalisés dans une maison d'édition, ils se sont révélés être pour certains un premier pas dans le milieu professionnel. Parallèles et plus fréquentes, d'autres formes d'apprentissage «sur le tas » sont mentionnées: du bénévolat pour un éditeur (gestion d'un forum, organisation de tournoi) ou encore des activités rémunérées d'animation à l'occasion de salons. Par leurs trajectoires, on comprend également que le métier initial et la formation scolaire se révèlent comme autant de dispositions acquises pour le métier. Issus du monde de la presse, de la publicité, de la vente, formés en marketing, en gestion, en école de commerce ou en métiers du livre, le jeu de société apparait comme un des prolongements possibles de leur diplôme. Pour deux des éditeurs, la culture ludique et la passion pour le jeu n'apparaissent d'ailleurs pas comme centrales dans leur insertion professionnelle en regard de leur formation. Pour eux, il s'agit moins d'une aventure personnelle dans un secteur "de niche" que d'un engagement professionnel dans des sociétés établies (depuis près d'un siècle) et importantes du secteur « de masse ». La passion apparait ici plus secondaire que la compétence à gérer et renouveler un catalogue composé de jeux anciens, « quasi-patrimoniaux ».

J'ai intégré la maison d'édition il y a à peu près trois ans, en tant que développeur, ce qui est l'équivalent d'un chef de projet chez un autre éditeur. Mon expérience a joué du fait de bien comprendre tous les systèmes et toutes les organisations, mais aussi d'avoir une dimension qui n'est pas seulement ludique, mais qui est plus... gestion, contrôle de gestion et gestion de projet. (Hector, éditeur) 


\section{Auteur et éditeur de jeu : de « vrais » métiers ?} fréquemment évoqué. Du côté des auteurs, la création de jeux est « un domaine qui n'a pas vraiment d'identité propre pour l'instant» comme le précise Franck. Pour cet enquêté et d'autres, le métier manque de visibilité et le statut d'auteur de jeu en France reste flou: les déclarations et les cotisations liées aux revenus issus de la création de jeux se révèlent souvent complexes ${ }^{27}$.

C'est vrai qu'on n'est pas reconnu, y'a pas de statut bien défini. Maintenant ça se passe dans une espèce d'indifférence générale et dans une ambiance plutôt bon enfant vis à vis de l'administration, Mais quelle que soit la manière dont on l'appelle, de toute manière, s'il y avait un statut et qu'on pouvait avoir quelque chose de plus défini, de plus cadré, ce serait pas plus mal. (Renaud, auteur).

Au-delà d'une clarification réglementaire, la revendication est aussi culturelle comme le précise Yannick : « un petit détail là, puisque tu vas continuer à faire des interviews, ce serait mieux que tu dises "auteur de jeu" plutôt que "créateur" parce que ça correspond à une sorte de combat qu'on a tous ensemble, pour nous faire reconnaitre comme des auteurs. Le recours fréquent à la notion "d'auteur", en lieu et place de celle de "créateur» ou «designer ", n'est pas anodin. L'usage du mot renvoie à un statut social plus légitime, par sa référence au monde des lettres et, à bien des égards, il en est proche. Le jeu de société renvoie en effet souvent à un " régime d'auctorialité " (Neeman, 2012). Les auteurs de jeux européens (allemands et français) bénéficient d'un statut particulier (et d'un droit d'auteur) et se voient reconnus comme les principaux créateurs. Ils sont mentionnés dans les règles, mais aussi sur les boites où leur nom apparait sous le titre comme une signature. En revanche, dans les pays anglo-saxons et dans le secteur de masse, la logique est différente et se rapproche davantage d'un « modèle industriel » que d'un « modèle auteuriste » (Daré, 2006) : les jeux sont signés par des équipes composées de «designers» moins souvent, voire rarement, mentionnées sur les boites. Parfois, mais pas toujours, ils sont «crédités» dans les règles. Il s'agit là d'une différence importante qui sépare le secteur entre un pôle que les acteurs considèrent comme marchand (qu'ils qualifient de "marché de masse ») et un pôle plus culturel (le « marché de niche » ou encore le « jeu moderne »).

Maintenant c'est acquis, sauf pour les grandes boites 殹sociétés殹 de jeux qui font du développement en interne. $\mathrm{MB}^{28}$ ou des trucs comme ça, y'aura pas le nom de l'auteur dessus, qui apparaît sur la boite. [...] Pour tous les jeux modernes, pour toutes les maisons d'édition maintenant, en France et en Allemagne en tout cas, c'est acquis [...] Ça me semble être la moindre des choses. Je suis très content de voir mon nom sur la boite. C'est moi qui l'ai fait, c'est moi qui ai eu l'idée, c'est moi qui ai donné naissance au jeu, c'est normal que mon travail et que mon nom soit mis dedans. (Sylvain, auteur)

Bien que majoritaire chez les enquêtés, cette revendication du statut d'auteur n'est pas unanimement partagée, et ce pour plusieurs raisons. D'une part, quelques-uns défendent le caractère contre-culturel du métier : «comme ça on peut faire ce qu'on veut » précise Richard. D'autre part, si nombre d'entre eux insistent sur la dimension créative de leur activité au même titre que d'autres formes d'art, plusieurs marquent cependant leur distance avec la notion auctoriale pour des raisons de légitimité culturelle. Sylvain préfère le terme de créateur : «je trouve qu'auteur de jeux a quand même un côté un peu, je dirais pas prétentieux, mais un peu ambitieux dans son ensemble. " Se référant à une vision parfois romantique et très «française » de 
l'écrivain tout entier dédié à son art (Sapiro et Rabot, 2017), sorte de " créateur incréé " (Bourdieu, 2002, p. 207), la différence entre la création littéraire et leur activité tient selon eux à la plus grande valeur sociale et culturelle des œuvres produites, à la difficulté du travail et aux compétences exigées ainsi qu'au mode de vie perçu, chez les écrivains, comme pleinement consacrée à l'écriture.

C'est pas comme un travail d'écrivain qui se met sur son roman. Ils s'astreignent à quelque chose de très régulier. Ceci dit, l'écriture, c'est tellement difficile que s'astreindre à ce genre de chose c'est utile. Pour le jeu de société, c'est quand même un petit peu plus facile, je dirai qu'on n'a pas vraiment l'angoisse de la page blanche, enfin moi je ne l'ai pas. (Franck, auteur)

Du côté des éditeurs, la problématique de la professionnalisation se pose différemment. Elle réside moins dans la perspective d'une reconnaissance culturelle que d'une clarification administrative. Si certains défendent la valeur sociale de leurs produits qui contraste avec la perception frivole et ludique de leur activité - «les gens pensent qu'on joue toute la journée " précise Anna - la plupart insiste sur le flou juridique de leur activité : «Déjà quand tu crées une boite, c'est pas clair pour le code NAF ${ }^{29}$. Nous on s'est mis en "fabricant de jouet et de jeux", mais t'en as qui sont en "éditeurs de livres" " (Sofien, éditeur). "Jeux et jouets ", » éditeurs de jeu vidéo ", " éditeurs de livres "... les statuts sont divers comme le précise un membre du syndicat des éditeurs des jeux de société : «Si on prend les éditeurs qui ne font que de l'édition de jeu, vous avez quasiment 20 codes différents ». Cette question de la reconnaissance administrative n'est pas seulement symbolique. L'enjeu est aussi de fixer un niveau de TVA commun. Les jeux produits par des éditeurs de livres jeunesse par exemple peuvent être commercialisés au taux de TVA de 5,5\% tandis que d'autres, selon le statut de la société et le réseau de diffusion, seront soumis au taux de $20 \%$. Il s'agit là d'un enjeu majeur pour les professionnels de l'édition, de « niche » ou de « masse ».

Qu'il s'agisse des auteurs ou des éditeurs, on perçoit donc dans les discours des enquêtés, sous une forme différente, mais proche, cette idée de " précarité identitaire " observée par Coulangeon (2004) chez les musiciens. Elle ne relève pas seulement d'un décalage entre des aspirations et la réalité des métiers, mais aussi d'une absence de visibilité, de reconnaissance institutionnelle et de professionnalisation du secteur. Oscillant entre deux revendications, culturelle d'un côté et réglementaire de l'autre, auteurs et éditeurs se rejoignent sur une demande commune d'organisation, de structuration et de reconnaissance. La création récente de deux associations professionnelles, l'union des éditeurs de jeux ${ }^{30}$ en 2016 et la société des auteurs de jeux de société en $2017^{31}$, marque explicitement ces préoccupations.

\section{Grandeurs et prestiges : les « grands » auteurs}

$\mathrm{Au}$ sein de ce monde du jeu, et comme dans tout monde social, se distribuent des "grandeurs ». Réputations, prestiges, renommées... quelques auteurs bénéficient d'un capital symbolique qui se traduit notamment par le qualificatif de «grand» auteur. De façon récurrente, apparait l'idée qu'il existe des professionnels à l'aune desquels certains des enquêtés se sentent souvent moins légitimes: "Je pense qu'en une heure l'entretien va être torché ; t'as pas 200000 questions à me poser non plus et puis c'est pareil les réponses, je suis pas [il cite deux « grands » auteurs] (Dominique, auteur). 
Parmi les critères de distinction entre " grands » et " petits » apparait en premier lieu le nombre de jeux vendus et la possibilité «d'en vivre». Les grands auteurs, selon Yannick, sont ceux qui « touchent beaucoup d'argent sur des jeux qui se vendent à des dizaines de milliers d'exemplaires par an ». En lien avec la question financière, le grand auteur se caractérise aussi par la taille de son catalogue qui peut lui permettre, à partir de ventes modestes, mais régulières, de constituer un capital et mener une activité d'auteur de façon continue.

Par exemple, Lui [un "grand» auteur], il a dû faire 50 jeux, et Lui [un autre] des centaines de jeux. Ce sont des gens qui sont en permanence en train de cogiter. Ils ont 100 prototypes qui se développent en même temps. Ils font évoluer tous leurs trucs et chaque année ils présentent leurs prototypes aux éditeurs. (Stéphane, auteur/éditeur)

41 La taille du catalogue est un critère discriminant à plusieurs titres. Il permet d'accumuler des ressources et témoigne dans le même temps d'une «durée dans le métier » que les acteurs, quelle que soit leur ancienneté, perçoivent toujours comme incertaine : "on ne sait jamais, ça va peut-être s'arrêter un jour » précise ainsi un auteur capé ${ }^{32}$. La grandeur dont témoigne le catalogue est plus élevée encore lorsqu'il comporte des succès de ventes, mais aussi des jeux primés au point d'être devenus des "références reconnues par les joueurs et les professionnels du milieu ludique comme étant de bons jeux » (Benoit, éditeur). La place qu'occupent les récompenses nationales et internationales est importante, nous le verrons, économiquement, mais aussi symboliquement.

Aux grands auteurs sont également associés des «styles » de création qui participent de leur renommée. Ils sont reconnaissables et recherchés pour une façon particulière de créer un jeu, de traiter un thème, ou de proposer une expérience ludique. Sont évoqués la "thématique asiatique d'Antoine Bauza ", "les jeux à deux de Bruno Cathala", les «délires de Roberto Fraga » «les jeux conviviaux de Bruno Faidutti» ou encore les mécaniques "implacables de Reiner Knizia». Plus encore, le qualificatif de "grand auteur » marque aussi la reconnaissance d'une autorité au point que, comme le précise Sylvain, lorsque l'un d'entre eux "frappe à la porte des maisons d'édition", il est davantage écouté qu'un autre. Plus sollicités, les "grands» auteurs sont aussi considérés comme plus aptes à fournir un jeu clef en main, prêt à être édité, sans que l'éditeur n'ait à intervenir de façon importante: «quand on a fait ces jeux avec ces deux auteurs, notre part de développement était beaucoup moins grande parce que ce sont deux grands, deux personnes qui ont énormément de bouteille. Qui savent faire des jeux de $\mathrm{A}$ à $\mathrm{Z}$, qui livrent un produit qui est fini. » (Gilles, éditeur).

A ces critères qualitatifs et quantitatifs s'ajoutent quelques compétences qui distinguent et témoignent d'une expertise dans le champ: la connaissance des différents éditeurs et de leur ligne éditoriale. Il s'agit en effet de connaitre l'ensemble des acteurs du domaine, mais aussi de savoir positionner un prototype auprès de la personne ad hoc. Sans réduire la question du «talent» (Menger 2002) au seul réseau d'interrelations de l'auteur, et sans l'évacuer non plus (DeNora 1995), le capital social accumulé et la capacité à le faire fonctionner de façon adaptée apparaissent comme la marque des « grands » professionnels.

Il y a quelques semaines il y a eu le salon le plus important pour moi de l'année, le salon de Nuremberg en Allemagne où j'ai été pour la 24e année. Et du coup, je prépare les maquettes, je prépare les rendez-vous, comme un vrai pro. J'ai des rendez-vous toutes les heures pendant cinq jours, et dans toutes les langues. Donc 
j'ai sélectionné des jeux en fonction des éditeurs, il faut être un peu pro si tu veux.

$\mathrm{Tu}$ ne peux pas montrer un jeu pour enfants à un éditeur qui ne fait pas ça.

(Renaud, auteur)

$\mathrm{Au}$ final, la grandeur d'un auteur se mesure de différentes manières : la taille de son catalogue, la durée dans le métier, les succès de vente, les récompenses, les sollicitations dont il est l'objet, la renommée dont il bénéficie, la reconnaissance d'un style, etc. L'accumulation de ces différents types de capitaux, sociaux et symboliques, est au principe des hiérarchies qui organisent ce monde, comme dans d'autres univers culturels et artistiques. Si cette opposition entre "grand » et «petit» est importante, les frontières sont en réalité très poreuses et fragiles. D'abord, les co-éditions entre un « amateur » et un " professionnel » sont fréquentes et les auteurs établis n'hésitent pas à tester, conseiller, voire à collaborer avec des "débutants" qui, de leur côté, perçoivent cet accompagnement comme une formation et un début de professionnalisation, à l'image des processus de cooptation et de guidage des nouveaux par les anciens dans la bande dessinée (Piette, 2015). Ensuite, la proximité entre amateurs et experts est d'autant plus étroite que l'effet de " petit monde » est renforcé par le développement des salons et des festivals dans lesquels, nous le verrons, auteurs et éditeurs, débutants et confirmés, de masse ou de niche, viennent présenter leur création en un même lieu.

\section{Les « gros » éditeurs : une économie symbolique des signatures}

Chez un éditeur comme moi, avoir un grand auteur, t'es tellement content de l'avoir que tu peux pas te permettre de le mettre un peu de côté. Chez un plus gros éditeur, qui en a déjà dans son catalogue ça sera un peu moins poussé » (Fabrice, auteur/éditeur)

Du côté des éditeurs, le prestige et le capital symbolique associés aux différentes maisons se révèlent tout aussi inégalement distribués. Cependant, à la différence des auteurs, on trouve moins fréquemment employé le terme de "grand» que celui de " gros ". Le sens métaphorique de cette épithète, qui renvoie à un poids plutôt qu'à une hauteur, est un premier indicateur de l'univers plus ouvertement compétitif du monde de l'édition. Si les auteurs évoquent souvent le travail de leur collègue sur un mode esthétique, les relations entre éditeurs sont plus explicitement décrites sur un mode concurrentiel.

À un premier niveau d'analyse, la valeur d'un "gros» éditeur s'évalue quantitativement: par son chiffre d'affaire, par la taille de son catalogue, par l'importance de ses équipes, par sa présence sur le marché et sa capacité à y déployer de nouveaux produits. Mais la dimension concurrentielle se manifeste plus particulièrement autour d'une économie des "signatures", autrement dit dans la capacité d'un éditeur à attirer des auteurs consacrés. Selon la logique de l'effet Matthieu $^{33}$ (Merton, 1968) ou celui du «winner-take-all» (Franck, Cook 1995), les "gros» éditeurs occupent une position plus favorable pour attirer les «grands» auteurs.

J'ai fait une proposition à un auteur très célèbre sur un de ses jeux. J'étais en concurrence avec un gros éditeur, et on avait les mêmes propositions financières, et les mêmes quantités en production. J'avais décidé de mettre le paquet [...] Eh ben, il a préféré le gros éditeur. Et il me l'a dit : "C'est pas contre toi, tu fais des très beaux 
jeux, tu te démènes sur les salons, on te voit, t'es sympa. Sauf que, y'a un gros éditeur qui nous proposait de le faire, on prend moins de risques en le faisant avec lui qu'avec toi. On l'aime pas plus que toi, on l'aime pas moins, mais on fait un choix concret quoi" ». (Fabrice, auteur/éditeur)

47 Face cette dynamique cyclique où auteurs et éditeurs alimentent respectivement la réputation de l'autre, il reste ainsi pour les jeunes professionnels de l'édition à mener un travail plus important de conviction auprès des auteurs réputés. Il s'agit d'attirer les vedettes, mais également de s'assurer de la qualité de la proposition. Le statut de jeune éditeur fait en effet courir le risque d'être un lieu d'édition par défaut d'un titre refusé ailleurs : "Quand, j'arrive à avoir des rendez-vous avec ces gens-là [les "grands » auteurs] ils me proposent des jeux qui ont été refusés par tous les autres. Des jeux de fin de queue, quoi, de fin de liste, tu le sens quand même ». (Fabrice, auteur/éditeur)

Si la taille de l'éditeur compte, il ne s'agit pas du seul critère. En effet, de façon comparable aux labels musicaux par exemple, la qualité attribuée à une maison est loin de se limiter à des dimensions strictement quantitatives ou économiques. Elle s'évalue aussi à partir de critères plus qualitatifs : un style d'édition, une ligne éditoriale, un travail graphique singulier... Comme le précise Sylvain, «les différents éditeurs sont connus pour avoir des jeux de bonne qualité matérielle, bien testés, équilibrés, violents, pas violents. On n'achète pas un jeu Filosofia ou un jeu Ystari au hasard. En l'achetant, on achète un jeu de cet éditeur » (Sylvain, éditeur).

Par une logique de spécialisation, des éditeurs développent ainsi une réputation dans certains domaines: "party games $»^{34}$, «jeux familiaux», " gros jeux $»^{35}$, «jeux coopératifs », etc. Si les éditeurs importants peuvent, comme dans le domaine des livres ou de la musique, s'assurer de leur présence dans l'ensemble du marché par le truchement des collections ou des gammes ${ }^{36}$, cette spécialisation peut être, pour un jeune éditeur, une façon d'exister à l'image de Gilles qui, au moment de l'entretien, hésite à publier un nouveau jeu, car il « dénote » de sa ligne éditoriale centrée sur les " jeux de stratégie rapide et conviviaux».

50 Cependant, le choix d'une maison d'édition par un auteur est loin d'être cynique et réalisé en parfait homo economicus. Il peut se faire selon des critères qui ne relèvent pas seulement d'un intérêt bien senti. Si les gros éditeurs restent bien sûr privilégiés, les auteurs, novices ou aguerris, choisissent aussi au regard d'une ligne éditoriale, d'un soutien à une société naissante, d'une opportunité, d'un "feeling", etc. Certains « grands auteurs » déclarent se sentir souvent plus concernés par le succès ou l'échec d'un jeu lorsqu'il est publié chez un éditeur naissant. Dans un entretien, Louis regrette le succès modeste d'un de ses titres édité dans une toute "petite boite " (deux personnes) qui a mis en danger la pérennité de l'entreprise. Son sentiment de responsabilité est moindre, précise-t-il, lorsqu'il s'agit d'entreprises importantes, plus à même d'amortir un échec, grâce au succès d'un ou plusieurs de ses jeux édités en leur sein.

51 Si l'économie des signatures est importante, elle ne définit donc pas l'ensemble du champ. L'effet Mathieu se voit pondéré. D’abord, parce qu'un « grand » auteur chez un "gros» éditeur ne signifie pas nécessairement un succès. Ensuite, parce que la reconnaissance symbolique, comme chez les écrivains (Sapiro et Rabot, 2017), n'est jamais pleinement acquise, elle est toujours tributaire du jeu à venir. Par ailleurs, en apparence tout au moins, le monde du jeu de société est un univers ouvert à tout nouvel entrant et tout nouveau "succès inattendu ». En comparaison avec d'autres 
univers culturels, que l'on pense au cinéma, à la musique ou au jeu vidéo, le cout d'entrée apparait en effet peu élevé aussi bien en termes de formations attendues ou exigées que d'un point de vue économique et matériel, toutes choses égales par ailleurs ${ }^{37}$. Cette accessibilité apparente est d'autant plus forte que les salons où l'on peut présenter des prototypes, rencontrer des éditeurs et des auteurs sont nombreux et que, dans le même temps, des concours, de prix et des récompenses, nationales internationales, encouragent les nouveaux entrants à « tenter leur chance».

\section{De l'importance des prix, des concours, des salons et des proto-zones} éditeurs de jeux déjà commercialisés, il existe des dispositifs de soutien plus spécifiques pour les aspirants auteurs. Comparable aux concours d'écriture dans le champ littéraire (Poliak 2006), des appels sont lancés dans la France entière, entre 20 et 30 par an. Parmi les plus anciens, on peut évoquer celui de Boulogne-Billancourt qui depuis 1977 récompense, soutient et encourage de jeunes créateurs dont certains ont par la suite fait « leur preuve». Sans toujours organiser des compétitions de ce type, les festivals occupent un rôle comparable dans cette logique de soutien en particulier avec des espaces consacrés aux amateurs pour y présenter leur prototype. Sous l'appellation parfois de "proto-zone», ces lieux sont visités par le public, mais aussi par les professionnels.

55 A certains égards, ces espaces se rapprochent du "simili-champ » que décrit Poliak (2006) dans son étude des écrivains amateurs, en tant que lieu distinct de l'univers des " vrais écrivains » et dont une des fonctions est d'entretenir une "croyance " en un accès possible au champ littéraire, mais aussi de « consoler » les perdants. Intégrées ou parallèles au "vrai salon», ces proto-zones, qui consacrent parfois le "meilleur prototype ", sont en effet des univers où les amateurs se réunissent entre eux et 
viennent tenter leur chance, espérant bénéficier de conseils et de remarques de professionnels, voire même un contrat d'édition. Cependant, la logique est différente de celle que décrit Poliak. D'abord parce que, même si ces lieux sont en effet constitués d' » aspirant auteurs ", ils sont dans les faits fréquentés par des auteurs qui ont déjà publié ${ }^{40}$. Certes, les auteurs prestigieux présentant leur création en cours sont rares dans ces espaces - ils rencontrent directement les éditeurs sur leur stand ou dans des espaces "professionnels "- mais ils les fréquentent. Les frontières sont très poreuses et l'étanchéité entre le sacré et le profane n'est pas aussi forte que dans le modèle du simili-champ. Les festivals, les concours et les proto-zones, même s'ils ne valent pas droit d'entrée, sont un important lieu de médiation entre publics, amateurs et professionnels. Comme l'observent Lahire et Bois à propos des écrivains, beaucoup d'auteurs de jeu même "réputés » sont en réalité passés par là (la majorité dans notre échantillon). Sans évacuer les illusions que peuvent entretenir ces dispositifs, leur fonction n'est pas seulement de confiner les aspirants aux marges du champ professionnel, mais aussi de les y former.

\section{Conclusion : champ et évolution du champ}

Depuis le milieu des années 1990, l'activité de création et d'édition de jeux de société s'est considérablement développée en France ${ }^{41}$. À ce jour, il est impossible de savoir si cette augmentation est durable ou s'il s'agit d'une «bulle» comme certains professionnels l'évoquent et le redoutent. S'il est difficile de prédire l'avenir économique du secteur, une évolution est cependant perceptible depuis les 40 dernières années : rattaché à la sphère du loisir et du divertissement familial, un secteur du jeu "adulte », aussi qualifié de » moderne ", s'est développé et autonomisé. Les professionnels interrogés, établis pour certains depuis plusieurs décennies, constatent tous cette transformation du secteur de même qu'une forme de légitimation culturelle, toute relative, mais nouvelle: invitations pour des "masters class", interviews dans la presse au-delà de la sphère spécialisée, multiplication des prix, etc. Le jeu de société semble ainsi connaitre une évolution comparable à celle de la bande dessinée qui, sous l'effet de générations nouvelles d'auteurs (Boltanski, 1975) et de la complexification de son lectorat (Berthou, 2011), est passée d'une pratique artistique déclassée à destination des enfants, à une pratique culturelle plus observée, étudiée et considérée (Piette, 2015).

Si le monde du jeu de société partage un ensemble de traits communs avec d'autres "monde de l'art», son appartenance au rang des «industries culturelles» (Moeglin, 2012) ou de "l'économie de la culture» (Benhamou, 2011) reste encore ambiguë. D'abord parce que le secteur du «divertissement familial » demeure nettement plus important en termes de publics et que les pratiques de passionnés sont bien plus «restreintes» (Berry, Coavoux et Ter Minassian, 2020). Ensuite, parce que sa considération par les pouvoirs publics est faible, et que leur intervention est quasiinexistante. À l'intérieur du champ, le jeu de société présente toutes les propriétés d'un monde culturel structuré autour d'une économie symbolique, faite de "grands jeux » et de "grands auteurs ». En revanche, à l'extérieur, il reste encore relativement invisible : le statut d'auteur, comme celui d'éditeur, n'est clairement pas identifié ou réglementé. Si celui-ci est défendu sur le mode de l'auctorialité par une partie importante des professionnels, il reste relativement bas dans l'échelle des hiérarchies culturelles, aux 
yeux notamment des auteurs, même réputés. Le jeu de société a ainsi la couleur d'un champ culturel sans en avoir ni la reconnaissance ni les outils institutionnels de professionnalisation comme pour la musique, la littérature ou encore, plus proche de lui et plus jeune, le jeu vidéo, secteur qui se révèle plus équipé en termes de syndicats, d'associations, de formations et de politiques publiques dédiées (Benghozi et Chantepie, 2017).

Nous avons fait le choix dans cet article d'une focalisation sur les auteurs et les éditeurs et nous n'avons que très partiellement évoqué l'organisation concrète de ce monde qui méritera d'être étudiée plus en détail, particulièrement en ce qui concerne les modes de relations, d'interactions et de coopérations entre les différents acteurs (Becker, 1988). Dans cet ensemble, auteurs et éditeurs sont bien sûr importants dans la construction économique et culturelle du secteur, mais il faut tout autant prendre en considération le rôle de la distribution, qui peut limiter ou non la diffusion d'un titre sur un territoire, et celui des magasins qui se révèlent être des lieux importants de médiation avec le public. Rappelons enfin que nous n'avons investigué qu'une partie de ce champ professionnel. A l'exception de quelques éditeurs et auteurs présents sur le marché de masse, le secteur que nous avons investigué est majoritairement celui des jeux "d'éditeurs " (Brougère, 1979) issus du secteur "de niche », autrement dit "un petit monde" dans le monde du jeu. Les fabricants de jeux traditionnels (dames, échecs, petits chevaux, cartes) et les acteurs du secteur de masse (Monopoly, Cluedo) restent encore méconnus et mériteraient un regard tout aussi attentif.

\section{BIBLIOGRAPHIE}

BECKER H. S. (1988), Les mondes de l'art, Paris, Flammarion.

BENGHOZI P.-J. et CHANTEPIE P. (2017), Jeux vidéo : L'industrie culturelle du XXIe siècle ?, Paris, Ministère de la Culture - DEPS.

BENHAMOU F. (2011), L'économie de la culture, Paris, La Découverte.

BERRY V. et BORZAKIAN M. (2015), « Les mondes du jeu ». RESET, n 4. https://doi.org/10.4000/ reset. 451

BERRY V., COAVOUX S. et TER MINASSIAN H. (2020), « Les Français et les jeux. Un état des lieux », in E. Duwez et P. Mercklé, Un panel français. L'Étude longitudinale par internet pour les sciences sociales, Paris, INED Éditions.

BERTHOU B. (2010), « La bande dessinée franco-belge : Quelle industrie culturelle ?», Textyles, $\mathrm{n}^{\circ}$ 36-37, p. 43-57.

BERTHOU B. (2011), « Les métamorphoses de la lecture de bande dessinée : Un défi pour la bibliothèque ", Bulletin des bibliothèques de France, tome 5, n 56, p. 36-39.

BIDET A. (2010), « Qu'est-ce que le vrai boulot ? Le cas d'un groupe de techniciens », Sociétés contemporaines, vol. 78, $\mathrm{n}^{\circ}$ 2, p. 115-135. 
BOLTANSKI L. (1975), « La constitution du champ de la bande dessinée », Actes de la recherche en sciences sociales, $\mathrm{n}^{\circ} 1, \mathrm{p}$. 37-59.

BOURDIEU P. (2002), Questions de sociologie, Paris, Les Éditions de Minuit.

BOURDIEU P. (2009), Les règles de l'art : Genèse et structure du champ littéraire, Paris, Éditions du Seuil.

BROUGÈRE G. (1979), « Du jouet industriel au jouet rationalisé », in R. Jaulin, Jeux et jouets, essai d'ethnotechnologie, Paris, Aubier.

BROUGÈRE G. (2008), « La ronde de la culture enfantine de masse », in G. Brougère, La ronde des jeux et des jouets, Paris, Autrement, p. 5-21.

BUSCATTO M. (2004), « De la vocation artistique au travail musical : Tensions, compromis et ambivalences chez les musiciens de jazz ", Sociologie de l'Art, vol. 5, n 3, p. 35-56.

COULANGEON P. (1999), « Les musiciens de jazz : Les chemins de la professionnalisation », Genèses, vol. 36, $n^{\circ} 1$, p. 54-68.

COULANGEON P. (2004), «L'expérience de la précarité dans les professions artistiques. Le cas des musiciens interprètes ", Sociologie de l'Art, vol. 5, n 3, p. 77-110.

DARRÉ Y. (2006), « Esquisse d'une sociologie du cinéma », Actes de la recherche en sciences sociales, $n^{\circ} 161-162$.

DELBOS G. \& JORION P. (1990), La transmission des savoirs, Paris, Édition de la Maison des Sciences de l'Homme.

DENORA, T. (1995), «Beethoven et l'invention du génie », Actes de la recherche en sciences sociales, $\mathrm{n}^{\circ} 110$, p. 36-45.

FRANK R. H. \& COOK P. J. (1996), The winner-take-all society : Why the few at the top get so much more than the rest of us, New-York, Penguin Books.

GROENSTEEN T. (1999), « La bande dessinée, entre production artisanale et diffusion de masse », in L. GERVEREAU (dir.), Peut-on apprendre à voir ?, Paris, ENSBA.

LAHIRE B. et BOIS G. (2006), La condition littéraire : La double vie des écrivains, Paris, La Découverte.

MENGER P.-M. (1983), Le paradoxe du musicien : Le compositeur, le mélomane et l'Etat dans la société contemporaine, Paris, Flammarion.

MENGER P.-M. (2002), Portrait de l'artiste en travailleur : Métamorphoses du capitalisme, Paris, Seuil.

MERTON R. K. (1968), « The Matthew effect $t$ », Science, vol. 159 n³810, p. 56-63.

MCEGLIN P. (2012), « Une théorie pour penser les industries culturelles et informationnelles ?» Revue française des sciences de l'information et de la communication, $\mathrm{n}^{\circ} 1$. https:// journals.openedition.org/rfsic/130

NEEMAN E. (2012), "Culture numérique et auctorialité : réflexions sur un bouleversement », $A$ contrario, $\mathrm{n}^{\circ} 17, \mathrm{p} .3-36$.

PAUGAM S. (2007), Le salarié de la précarité : Les nouvelles formes de l'intégration professionnelle, Paris, PUF.

PIETTE J.-E. (2015), « L'accession au statut d'artiste des dessinateurs de bande dessinée en France et en Belgique ", Sociologie de l'Art, n²3-24, p. 111-128.

POLIAK C. F. (2006). Aux frontières du champ littéraire : Sociologie des écrivains amateurs, Paris, Economica. 
S.A.J. [Société des Auteurs de Jeux] (2019), « Résultats de la consultation des auteurs de jeux de société réalisé en 2018 ». http://www.societedesauteursdejeux.fr/wp-content/uploads/2019/07/ CONSULTATION_AUTEURS_JDS.pdf

SAPIRO G. et RABOT C. (2017), Profession ? Écrivain, Paris, CNRS éditions.

STIÉNON V. (2008), « Des "univers de consolation". Note sur la sociologie des écrivains amateurs », COnTEXTES, Notes de lecture $:$ http://journals.openedition.org/contextes/2933

\section{NOTES}

1. Taxe à la Valeur Ajoutée qui s'applique selon des taux différents à tous les produits commercialisés au sein de l'Union Européenne

2. Voir à ce titre les travaux de Vinciane Zabban et Hovig Ter Minassian dans le cadre du projet de recherche TETRIS : https://tetris.hypotheses.org/author/tetris

3. Il s'agit de personnes ayant au moins un jeu publié par un éditeur et distribué en magasin ou en ligne.

4. Dans notre échantillon, huit de nos enquêtés ont une double activité : auteur et éditeur. L'un d'entre eux est auteur et illustrateur (pour des jeux autres que les siens). Pour ces enquêtés, une facette de leur activité a été plus explorée que l'autre.

5. Des acteurs peuvent se spécialiser dans la distribution, le financement de projet, la mise en forme commerciale (packaging), le développement de la presse. Des éditeurs peuvent mener plusieurs de ces activités simultanément. Nous avons gardé ici les professionnels dont l'activité principale est décrite comme relevant de l'édition (développements de jeux).

6. Nous nous sommes également appuyés sur des entretiens donnés par certains des auteurs pour la presse spécialisée : les sites Ludovox et Tric-Trac et le podcast Proxi-Jeux.

7. Union des Editeurs du Jeu de société [http://www.uej.fr], presse économique [https:// www.lesechos.fr/industrie-services/conso-distribution/le-marche-francais-du-jeu-de-societe-seprofessionnalise-993882], presse spécialisée [ https://bibliojeu.net/editions/] et discours professionnels

8. Les sociétés d'édition peuvent appartenir à des groupes plus grands. Dans le cas d'un éditeur allemand dont nous avons interrogé un salarié de la filiale française (une trentaine de salariés), la société mère regroupe plus de 1000 salariés dans le monde. De la même façon, des petites maisons d'édition, aux équipes réduites, peuvent appartenir à un groupe plus important, comme le groupe français Asmodée qui regroupe plusieurs « studios » pour près de 200 salariés en France et plus de 600 dans le monde [sites et données de l'éditeur : https://corporate.asmodee.com/ about-us/ et https://fr.asmodee.com/fr/about-asmodee/].

9. Elle regroupe des auteurs du secteur et milite pour la reconnaissance du métier. Elle a diffusé en 2018 une enquête auprès des auteurs de jeux $(n=86)$ francophones (93\% résident en France) ayant publié au moins un jeu depuis le 1er janvier 2015 (autoédition exclue).

10. Notre échantillon se révèle cependant très proche des données professionnelles fournies par l'UEJ et par la SAJ (2019).

11. L'enquête de la SAJ (2019) estime à $5 \%$ la proportion d'autrices

12. Les données recueillies sont relativement proches de l'enquête de la SAJ (2019) en termes d'âge et de formation. Près de $70 \%$ des auteurs ont au moins un diplôme de niveau $3(\mathrm{bac}+3)$ : $31 \%$ ont un diplôme de niveau de bac +3 et $38 \%$ de niveau bac +5 .

13. Dans l'enquête de la SAJ (2019), la moitié des auteurs déclarent avoir publié entre 1 et 3 jeux, près d'un quart entre 4 et 9 jeux, et plus de 10 jeux pour le dernier quart. 
14. Les données sont proches de celles de l'enquête de la SAJ (2019). Plus de $80 \%$ des auteurs ont un deuxième métier : $30 \%$ sont cadre en entreprise, dans la fonction publique ou en profession libérale, $15 \%$ sont enseignants.

15. Nombreux sont les écrivains qui ont un second métier souvent lié à l'écriture : enseignement, édition, traduction, ateliers, etc.

16. Les musiciens de jazz donnent souvent des cours, jouent dans des orchestres sur des registres différents (rock, variété ou musique pour enfants), dans le cadre d'animations commerciales ou de sessions privées ou encore composent des musiques pour la publicité, la télévision ou le cinéma.

17. En parallèle à un travail souhaité, désiré et personnel, les dessinateurs travaillent souvent dans l'illustration de presse ou la publicité.

18. Les noms des enquêtés ont été changés pour respecter leur anonymat.

19. Les redevances (royalties) sont négociées selon la notoriété et l'ancienneté des deux parties. Dans notre échantillon, elles oscillent entre 3 et $8 \%$ sur le prix de vente professionnel. Cette logique est confirmée par l'enquête de la SAJ (2019): ce type de contrat concerne $74 \%$ des auteurs interrogés. Des avances sur droits sont souvent versées : $65 \%$ des auteurs déclarent en avoir reçu et dans $75 \%$ des cas, elles s'élèvent à moins de 2000 euros.

20. $20 \%$ estimaient les droits d'auteur perçus l'année précédente entre « 5000 et 20000 euros » et $18 \%$ à « 20000 euros et plus ». (SAJ 2019)

21. Répertoire Nationale des Certifications Professionnelles

22. Cette logique est confirmée dans l'enquête de la SAJ (2019) : la majorité (68\%) des auteurs ayant un second métier (autre que le jeu) déclarent que leur activité (et leur situation) professionnelle leur convient. $32 \%$ souhaitent travailler comme auteur à plein temps ou plus généralement dans le monde de l'édition de jeux.

23. Les noms des enquêtés ont été changés pour respecter leur anonymat.

24. La logique est différente chez les musiciens classiques où « les pratiques amateurs sont assez nettement déconnectées des pratiques professionnelles» (Coulangeon, 2004).

25. Utilisant des dispositifs numériques, à l'image du jeu Unlock (Space Cowboys, 2017)

26. «Faiseur de roi ». Terme qui dans le monde du jeu désigne les titres dans lesquels une ou plusieurs actions d'un joueur décideront entre plusieurs adversaires de celui qui remportera la partie.

27. Dans l'enquête de la SAJ (2019), $45 \%$ des auteurs déclarent leur revenu aux impôts en «traitements et salaires », $29 \%$ en " entreprise individuelle », et $23 \%$ ne les déclarent pas (les revenus souvent très marginaux, et l'enquête inclut $7 \%$ d'auteurs dont la résidence fiscale n'est pas en France). Concernant les cotisations sociales issues de ces revenus, $52 \%$ n'en versent pas, $23 \%$ en versent à la sécurité Sociale des auteurs (AGESSA/MDA), $9 \%$ à l'URSSAF, $1 \%$ via l'éditeur (SAJ 2019).

28. Initiale de Milton Bradley, célèbre éditeur de jeux nord-américain : Yahtzee, Docteur Maboul, Twister, Destin, Puissance 4, Qui-est-ce ?... Créé en 1860, il appartient depuis 1984 au groupe Hasbro, fabricant mondial de jeux et de jouets.

29. Nomenclature d'Activité Française

30. Il regroupe près d'une cinquantaine d'éditeurs, de petite et moyenne taille, mais quelques éditeurs plus importants y participent

31. L'association regroupe près d'une trentaine d'auteurs.

32. Dans l'enquête de la SAJ (2019), plus de $70 \%$ des auteurs interrogés se déclarent pas (48\%) ou peu (23\%) « confiant[s] sur le fait de vivre pleinement de [ses] activités d'auteur dans les années à venir ».

33. Effet de renforcement, voire de redoublement d'une grandeur sur une autre, initialement mis en évidence dans le monde de la recherche scientifique. 
34. Termes qui dans le monde du jeu désignent des jeux courts, rapides et à interactions fréquentes entre joueurs, à l'image du jeu Time's Up (Repos Production, 2005). Proche de cette catégorie, certains évoquent les " apéro jeux ».

35. Pour désigner des jeux longs (plusieurs heures), supposant un matériel important et des règles denses, à l'image des jeux Civilization (1981, Avalon Hill), Great Western (Gigamic, 2017), Through the Ages (IELLO, 2010).

36. Ou encore par le rachat d'autres éditeurs.

37. La conception d'un prototype de jeu, qui suppose le plus souvent des pions, des cartes et des dés se révèle en effet moins couteuse que la conception d'une maquette en musique ou dans le jeu vidéo qui suppose un équipement plus conséquent.

38. Il s'agit parfois, mais pas toujours, de parodier les cérémonies littéraires ou cinématographiques, comme lors de la cérémonie des prix de l'As d'or 2020. La vidéo est disponible à l'adresse : https://www.youtube.com/watch ?v =tUkiHXKi_fM

39. Les sites spécialisés rapportent régulièrement les résultats du concours qui se voient commentés. Voir à titre d'exemple, Tric-Trac [https://www.trictrac.net/actus/et-le-kennerspiel-des-jahres-est] ou Ludovox [https://ludovox.fr/le-spiel-des-jahres-2018-la-selection]

40. Dans l'enquête de la SAJ (2019), plus de $85 \%$ des auteurs déclarent fréquenter les festivals dans l'année : $17 \%$ de façon au moins mensuelle, $30 \%$ au moins une fois par trimestre et $41 \%$ une ou deux fois par an. Ils le font le plus souvent à leur frais.

41. Estimé à plus de 300 millions en 2018 [source : NPD group].

\section{RÉSUMÉS}

Cet article étudie le monde des éditeurs et des auteurs de jeux de société en France. Après avoir rappelé les conditions de l'enquête, basée sur une série d'entretiens auprès de professionnels du secteur $(n=35)$, il s'intéresse aux trajectoires sociales et scolaires des éditeurs et des auteurs de jeux. A travers l'étude de leur carrière, il s'agit d'analyser la façon dont ils se professionnalisent. Dans un deuxième temps, l'article interroge l'organisation du secteur en le comparant à d'autres domaines culturels ou artistiques (littérature, cinéma, bande dessinée). Si le monde du jeu de société présente tous les signes d'un «monde de l'art» (division du travail spécifique et importance du capital symbolique sous forme de "prix »), sa reconnaissance et son inscription dans le domaine culturel demeurent ambiguës. Malgré une demande de reconnaissance culturelle et institutionnelle de leur activité, le soutien des pouvoirs publics et des instances de légitimation reste quasi-inexistant.

This article studies the world of board game publishers and authors in France. After recalling the conditions of the survey, based on a series of interviews with professionals in the sector $(n=35)$, it looks at the social and educational trajectories of game publishers and authors. Through the study of their careers, the aim is to analyze the way in which they professionalize themselves. Secondly, the article questions the organization of the sector by comparing it with other cultural or artistic fields (literature, cinema, comics). Though the world of board games presents all the signs of an "art world" (specific division of labour and importance of symbolic capital in the form of "prizes"), its recognition and inscription in the cultural domain remains ambiguous. Despite a demand for cultural and institutional recognition of their activity, the support of public authorities and legitimizing bodies remains almost non-existent. 
INDEX

Mots-clés : jeu de société, organisation, professionnalisation, monde de l'art

Keywords : board game, organization, professionalization, art world

\section{AUTEURS}

VINCENT BERRY

EXPERICE, Labex ICCA, Université Sorbonne Paris Nord

NATHALIE ROUCOUS

EXPERICE, Université Sorbonne Paris Nord 